\title{
Chemerin as a marker of subclinical cardiac involvement in psoriatic patients
}

\author{
Feyza Aksu ${ }^{1}$, Mustafa Caliskan ${ }^{1}$, Nursen Keles ${ }^{1}$, Aybala Erek Toprak ${ }^{2}$, \\ Tugba Kevser Uzuncakmak ${ }^{3}$, Osman Kostek ${ }^{4}$, Yusuf Yilmaz ${ }^{1}$, Kenan Demircioglu ${ }^{1}$, \\ Esad Cekin ${ }^{1}$ Ibrahim Ozturk ${ }^{2}$, Ayse Serap Karadag ${ }^{3}$ \\ ${ }^{1}$ Department of Cardiology, Faculty of Medicine, Istanbul Medeniyet University, \\ Goztepe Training and Research Hospital, Istanbul, Turkey \\ ${ }^{2}$ Department of Biochemistry, Faculty of Medicine, Istanbul Medeniyet University, \\ Goztepe Training and Research Hospital, Istanbul, Turkey \\ ${ }^{3}$ Department of Dermatology, Faculty of Medicine, Istanbul Medeniyet University, \\ Goztepe Training and Research Hospital, Istanbul, Turkey \\ ${ }^{4}$ Department of Internal Medicine, Faculty of Medicine, Istanbul Medeniyet University, \\ Goztepe Training and Research Hospital, Istanbul, Turkey
}

\begin{abstract}
Background: Chemerin has been associated with psoriasis and inflammation, but there are no studies demonstrating an association between chemerin and subclinical cardiac involvement in psoriatic patients. Therefore, the present study aimed to evaluate whether psoriatic patients with increased epicardial fat tissue, impaired flow-mediated dilatation, and diastolic dysfunction have higher serum chemerin levels than a healthy control group.

Methods: The study included 60 psoriatic patients and 32 healthy controls. Echocardiographic parameters, epicardial fat tissue, flow-mediated dilatation, and chemerin levels were recorded for both groups. Results: The serum levels of chemerin in the psoriatic patients were significantly higher than in the control group. The diastolic function parameters, including isovolumic contraction and relaxation time, E'/A' (early diastolic mitral annular velocity/late diastolic mitral annular velocity), and E/E' (early diastolic peak velocity of mitral inflow/early diastolic mitral annular velocity) values, differed significantly between the groups. Epicardial fat tissue was significantly higher and flow-mediated dilatation was significantly lower in psoriatic patients than in the controls. Chemerin was significantly positively correlated with age, body mass index, systolic and diastolic blood pressures, waist circumference, $E / E^{\prime}$, and epicardial fat tissue. Serum chemerin was significantly negatively correlated with $E^{\prime}, E^{\prime} / A^{\prime}$, and flow-mediated dilatation. A multiple linear regression analysis showed that chemerin was independently correlated with $E / E$ '.

Conclusions: Psoriatic patients exhibit early subclinical atherosclerosis and diastolic dysfunction. Chemerin can be used as a marker to screen for patients with subclinical cardiac involvement. (Cardiol J 2017; 24, 3: 276-283)
\end{abstract}

Key words: chemerin, flow-mediated dilatation, epicardial fat tissue, psoriasis

Address for correspondence: Feyza Aksu, MD, Department of Cardiology, Istanbul Medeniyet University, Goztepe Training and Research Hospital, 34730 Istanbul, Turkey, tel: +90 216-566-40-14, fax: +90 216-566-40-00, e-mail: feyzaulusoyaksu@yahoo.com

Received: 26.12.2016 Accepted: 26.01.2017 


\section{Introduction}

Chemerin, a novel chemokine that modulates activation and chemotaxis of dendritic cells, macrophages, and neutrophils, is highly expressed in the liver and white adipose tissue [1]. In addition to its immunomodulatory effects, associations have been reported between chemerin and components of metabolic syndrome, including body mass index (BMI), plasma triglyceride (TG) levels, and hypertension [1, 2]. Recently, strong chemerin expression and increased plasmacytoid dendritic cells have been associated with psoriatic skin [3]. Psoriasis is a chronic inflammatory disease of the skin, affecting 2-3\% of the world's population [4]. It is known that psoriasis is associated with earlier development of atherosclerosis and a higher prevalence of subclinical left ventricular (LV) dysfunction, even in those without traditional cardiovascular $(\mathrm{CV})$ risk factors [5]. The main mechanism behind these conditions is the chronic inflammatory process of psoriasis $[6,7]$. In light of these findings, chemerin, which plays a role in psoriasis pathogenesis and has immunomodulatory effects, may be useful as an early marker of subclinical CV disease (CVD) in patients with psoriasis.

In chronic inflammatory diseases, including psoriasis and systemic lupus erythematosus, subclinical myocardial disease is identified with tissue Doppler imaging (TDI), a modified Doppler technique that enables direct measurement of myocardial contractile and relaxation velocities, with high reproducibility and ease of performance [8-10]. Measuring flowmediated dilatation (FMD) is a noninvasive method of detecting endothelial dysfunction, which is regarded as an early stage of atherosclerosis [11]. Epicardial fat tissue (EFT) is considered a metabolically active endocrine organ that strongly influences both the formation and advancement of coronary artery disease [12-14].

Recent trials involving psoriatic patients have demonstrated that increased EFT and impaired FMD are related to increased risk of atherosclerosis $[15,16]$. However, there are no data on the association between serum chemerin levels and atherosclerosis risk and subclinical cardiac involvement in psoriatic patients.

The present study hypothesized that the serum chemerin level may be a potential novel early marker of atherosclerosis and subclinical myocardial dysfunction in patients with psoriasis. Therefore, the study aimed to evaluate whether psoriatic patients with increased EFT, impaired FMD, and diastolic dysfunction on TDI also have higher serum chemerin levels than a healthy control group.

\section{Methods}

\section{Study population}

The study enrolled consecutive patients with psoriasis who attended the outpatient clinic of the Dermatology Unit, Istanbul Medeniyet University, Faculty of Medicine, Goztepe Training and Research Hospital, Istanbul, Turkey, between January 2014 and January 2015. These patients were compared with healthy volunteers. The exclusion criteria were age $>60$ years, smoking, the presence of structural heart disease, systemic diseases indicating cardiac involvement (e.g., hypertension, hypo- or hyperthyroidism, diabetes mellitus, collagenosis, or renal failure), CVD (e.g., prior myocardial infarctions, peripheral artery disease, or stroke), dyslipidemia, other dermatologic diseases affecting the immune system, psoriatic arthritis, and use of biologic agents [17]. Also excluded were patients with left ventricular mass index (LVMI) of $\geq 125 \mathrm{~g} / \mathrm{m}^{2}$ for men or $\geq 110 \mathrm{~g} / \mathrm{m}^{2}$ for women, or a BMI of more than $35 \mathrm{~kg} / \mathrm{m}^{2}$. Written informed consent was obtained from all patients before enrollment. The study protocol was approved by the institutional ethics committee, and the study was conducted according to the principles of the Declaration of Helsinki.

\section{Biochemical and clinical assessment}

Measurements included total cholesterol, low-density lipoprotein cholesterol (LDL-C), high-density lipoprotein cholesterol (HDL-C), TG, glucose, and high-sensitivity C-reactive protein (hs-CRP) levels. BMI, waist circumference, systolic blood pressure (SBP), and diastolic blood pressure (DBP) were recorded according to standardized procedures. The extent of skin involvement was assessed using the Psoriasis Area and Severity Index (PASI) [18].

\section{Chemerin measurement}

To analyze the concentration of chemerin levels, commercial ELISA kits (Biovendor; Czech Republic) were used according to the manufacturer's instructions. The intra-assay coefficient of variations $(\mathrm{CoVs})$ of the chemerin assay were $5.1 \%$ at $177.5 \mathrm{ng} / \mathrm{mL}$ and $7 \%$ at $263.6 \mathrm{ng} / \mathrm{mL}$. The inter-assay CoVs of the chemerin assay were $8.3 \%$ at $140.6 \mathrm{ng} / \mathrm{mL}$ and $6.9 \%$ at $222.7 \mathrm{ng} / \mathrm{mL}$. The assay sensitivity was $0.1 \mathrm{ng} / \mathrm{mL}$ and the upper limit of the standard was $8 \mathrm{ng} / \mathrm{mL}$. Measurements were 
taken using an ELISA plate reader (Multiscan Go; Thermo Fisher Scientific, Inc.; Waltham, MA, USA) and a plate washer (Thermo Fisher Scientific, Inc.). Each well absorbance was determined at $450 \mathrm{~nm}$ by plotting the mean absorbance $(\mathrm{Y})$ of standards against the known concentration (X) of standards on alogarithmic scale. The results were reported as the concentration of chemerin $(\mathrm{ng} / \mathrm{mL})$ in the samples.

\section{Echocardiographic evaluation}

Echocardiographic evaluation of the study population was conducted with transthoracic echocardiography using a GE Medical Systems VIVID 3 SyncMaster 591S machine. M-mode, 2-dimensional, and finally both standard and pulsed tissue Doppler echocardiographic evaluations were performed with participants in the lateral decubitus position. Recommendations of the American Society of Echocardiography were followed while performing the echocardiographic measurements. Echocardiography determined the following parameters: diastolic interventricular septal (IVS) and posterior wall (PW) thickness, left atrium (LA) diameter, $\mathrm{LV}$ end-systolic diameter (LVSD) and LV end-diastolic diameter (LVDD), ejection fraction (EF), late diastolic peak velocity of mitral inflow (A), early diastolic peak velocity of mitral inflow (E), E/A ratio, deceleration time (DT), isovolumic contraction time (IVCT) and relaxation time (IVRT), ejection time (ET), late diastolic mitral annular velocity (A'), early diastolic mitral annular velocity (E'), E'/A' ratio, E/E' ratio, mitral annular systolic motion velocity (Sm), and mitral annular ejection time (ET'). The EFT was defined as the hypoechoic space on the right ventricular free wall on the parasternal long-axis view [19].

\section{FMD measurement}

Brachial artery measurements were taken by a qualified cardiologist who was blinded to previously obtained data. Ultrasonographic scanning was performed using high-resolution B-mode equipment (Aplio XU; Toshiba; Glen Mills, PA, USA) with a $7.5-\mathrm{MHz}$ linear transducer. The patients were examined in the morning, after fasting for $8 \mathrm{~h}$ with no alcohol or caffeine, and after $15 \mathrm{~min}$ of rest. The right arm was relaxed and placed in an extended position for analysis of the brachial artery $3-5 \mathrm{~cm}$ above the antecubital fossa. Arm position was not changed throughout the study, and the point on the arm at which an acceptable image had been obtained was marked for repeated use. A pneumatic tourniquet was placed approximately $3-5 \mathrm{~cm}$ above antecubital fossa, and $200 \mathrm{~mm} \mathrm{Hg}$ of pressure was applied for $5 \mathrm{~min}$. A second ultrasonographic scan was taken after sudden deflation of the cuff. The average of measurements taken at 30,60, and $90 \mathrm{~s}$ was calculated and used. The diameter change caused by FMD was expressed as the percentage change relative to that on initial resting scan [20].

\section{Statistical analyses}

All calculations were performed using the statistical software package SPSS 9 for Windows (SPSS; Chicago, IL, USA). The Kolmogorov-Smirnov test was used to determine distribution of values, whether normal or not. Descriptive analyses were performed using mean and standard deviation for normally distributed variables, and medians and interquartile ranges for non-normally distributed variables. Either the Student's t-test or the MannWhitney U-test were used to compare parameters between groups, as appropriate. Pearson's correlation or Spearman's rho correlation analyses were used to test possible associations between chemerin and the study variables, as appropriate. Multivariate linear regression models explored whether the association between chemerin and E/E' was independent of age, SBP, DBP, waist circumference, glucose, and LDL-C levels. $\mathrm{P}<0.05$ was considered as statistically meaningful.

\section{Results}

This study used data from 60 psoriatic patients (mean age $44 \pm 14$ years; 23 women, 37 men) and 32 control subjects (mean age $42 \pm 8$ years; 16 women, 16 men). Neither age nor sex varied significantly between the two groups ( $p>0.05$ ). In addition, there were no statistically significant differences in BMI, waist circumference, SBP, or DBP between the two groups (Table 1 ).

The serum levels of chemerin in the psoriatic patients were significantly higher than in the control group $(332 \pm 73 \mathrm{ng} / \mathrm{mL}$ vs. $301 \pm 60 \mathrm{ng} / \mathrm{mL}$; $\mathrm{p}=0.04)$. The HDL-C values of the psoriatic patients were observed to be significantly lower than those of the controls $(43 \pm 12 \mathrm{mg} / \mathrm{dL}$ vs. $53 \pm 15 \mathrm{mg} / \mathrm{dL}$; $\mathrm{p}=0.003)$. All other biochemical parameters of the psoriatic patients were similar to those of the control group (Table 1).

All patients had normal IVS thickness, PW thickness, and LVDD, LVSD, and EF measurements, and there were no significant differences between the groups in IVS thickness, LVDD, LVSD, LA diameter, and EF. However, the PW thickness of the psoriatic patients was significantly greater 
Table 1. Demographic characteristics and laboratory variables.

\begin{tabular}{|c|c|c|c|}
\hline & $\begin{array}{l}\text { Psoriasis subjects } \\
\qquad(\mathrm{n}=60)\end{array}$ & $\begin{array}{l}\text { Control subjects } \\
\qquad(n=32)\end{array}$ & $\mathbf{p}$ \\
\hline Age [years] & $44 \pm 14$ & $42 \pm 8$ & 0.67 \\
\hline Gender: female/male & $23 / 37$ & $16 / 16$ & 0.28 \\
\hline Psoriasis Area and Severity Index & $9.89 \pm 6.93$ & & \\
\hline BMI $\left[\mathrm{kg} / \mathrm{m}^{2}\right]$ & $27.2 \pm 4.5$ & $27.8 \pm 4.4$ & 0.56 \\
\hline Systolic BP [mm Hg] & $120(120-135)$ & $127.5(120-139.7)$ & 0.29 \\
\hline Diastolic BP [mm Hg] & $80(70-85)$ & $79(70.2-80)$ & 0.52 \\
\hline Glucose $[\mathrm{mg} / \mathrm{dL}]$ & 95 (89.5-107.5) & $92(89.2-96.7)$ & 0.14 \\
\hline Total cholesterol [mg/dL] & $194 \pm 42$ & $211 \pm 50$ & 0.1 \\
\hline LDL-C [mg/dL] & $122 \pm 35$ & $128 \pm 40$ & 0.5 \\
\hline $\mathrm{HDL}-\mathrm{C}[\mathrm{mg} / \mathrm{dL}]$ & $43 \pm 12$ & $53 \pm 15$ & 0.003 \\
\hline Triglyceride $[\mathrm{mg} / \mathrm{dL}]$ & $123(81-171)$ & 99.5 (71.2-179.7) & 0.27 \\
\hline hs-CRP [mg/dL] & $0.99 \pm 1.47$ & $0.33 \pm 0.27$ & 0.008 \\
\hline Waist circumference $[\mathrm{cm}]$ & $97 \pm 12$ & $98 \pm 13$ & 0.79 \\
\hline Chemerin $[\mathrm{ng} / \mathrm{mL}]$ & $332 \pm 73$ & $301 \pm 60$ & 0.04 \\
\hline
\end{tabular}

Data are mean \pm standard deviation $(\mathrm{SD})$ or median interquartile range (IQR). $\mathrm{F}$ - female; $\mathrm{M}$ - man; BMI — body mass index; $\mathrm{BP}$ - blood pressure; hs-CRP — highly sensitive C-reactive protein; HDL-C — high-density lipoprotein cholesterol; LDL-C — low-density lipoprotein cholesterol

than that of the control group $(9 \pm 1.2 \mathrm{~mm}$ vs. $8 \pm 0.9 \mathrm{~mm} ; \mathrm{p}=0.01$ ).

In addition, the diastolic function parameters, including IVRT, E'/A', and E/E' values, differed significantly between the groups and were found to be impaired in psoriatic patients (Table 2). Furthermore, EFT values of psoriatic patients were significantly higher than those of controls $(0.33 \pm$ $\pm 0.13 \mathrm{~cm}$ vs. $0.25 \pm 0.12 \mathrm{~cm} ; \mathrm{p}=0.04)$. However, the percentage of FMD was significantly lower in psoriatic patients than in the controls $(6.9 \pm 2.7 \%$ vs. $11.8 \pm 3.3 \%$; $\mathrm{p}<0.001$ ).

A correlation analysis was conducted for the correlations between serum chemerin level and age, BMI, waist circumference, PASI score, SBP, DBP, total cholesterol, TG, LDL-C and HDL-C, glucose, hs-CRP, EFT, FMD, and the echocardiographic parameters. Chemerin was significantly positively correlated with age, BMI, SBP, DBP, waist circumference, E/E', and EFT. Serum chemerin was significantly negatively correlated with E', E'/A', and FMD (Table 3). No significant correlation was found between serum chemerin and other study variables.

A multiple linear regression analysis with $\mathrm{E} / \mathrm{E}^{\prime}$ as the dependent covariate, and with other study variablesas independent covariates, including age, SBP, DBP, waist circumference, glucose, and LDL-C levels, showed that chemerin was independently correlated with E/E' (Table 4).

\section{Discussion}

The present study demonstrated that serum chemerin levels, which are associated with the production of pro-inflammatory mediators, are significantly higher in psoriatic patients than in the healthy population. The results also showed that serum chemerin levels directly correlate with the predictors of subclinical atherosclerosis: increased EFT and impaired FMD and LV diastolic function parameters. In addition, serum chemerin levels are strongly associated with subclinical cardiac dysfunctionin psoriatic patients.

Psoriasis is a chronic inflammatory skin disease characterized by well-marked, erythematous, scaling plaques on the extremities and trunk [4]. Psoriasis not only negatively affects the patient's quality of life, but its chronic inflammation also increases the risk of $\mathrm{CV}$ morbidity and mortality [21]. However, it is not only inflammation that could justify this association; recent evidence also suggests a role for autonomic CV system dysregulation [22]. Therefore, early detection of subclinical atherosclerosis in patients with psoriasis would aid in reducing $\mathrm{CV}$ morbidity and mortality.

Chemerin has been described as a pro-inflammatory adipokine that is released by the white adipose tissue itself, and its serum levels have been found to be strongly associated with components of metabolic syndrome, including elevated BMI, TG, 
Table 2. Echocardiographic parameters.

\begin{tabular}{|c|c|c|c|}
\hline & Control subjects $(n=32)$ & Psoriasis subjects ( $n=60$ ) & $\mathbf{P}$ \\
\hline $\mathrm{LA}[\mathrm{mm}]$ & $35 \pm 4$ & $34 \pm 4$ & 0.38 \\
\hline IVS [mm] & $9(8-10)$ & $9(9-10)$ & 0.05 \\
\hline $\mathrm{PW}[\mathrm{mm}]$ & $8(7-8.5)$ & $9(7-8.5)$ & 0.01 \\
\hline LVEDD [mm] & $49 \pm 4$ & $48 \pm 4$ & 0.25 \\
\hline LVESD [mm] & $31 \pm 4$ & $30 \pm 3$ & 0.07 \\
\hline EF [\%] & $65 \pm 4$ & $66 \pm 4$ & 0.18 \\
\hline$E[\mathrm{~m} / \mathrm{s}]$ & $0.69 \pm 0.11$ & $0.71 \pm 0.14$ & 0.74 \\
\hline $\mathrm{A}[\mathrm{m} / \mathrm{s}]$ & $0.54 \pm 0.09$ & $0.58 \pm 0.14$ & 0.14 \\
\hline DT [ms] & $208(192.5-256)$ & 209 (175-257.5) & 0.6 \\
\hline$E / A$ & $1.3 \pm 0.3$ & $1.2 \pm 0.4$ & 0.6 \\
\hline IVRT [ms] & $87 \pm 14$ & $79 \pm 14$ & 0.02 \\
\hline IVCT [ms] & $70 \pm 13$ & $71 \pm 13$ & 0.7 \\
\hline $\mathrm{ET}[\mathrm{ms}]$ & $264 \pm 28$ & $276 \pm 22$ & 0.07 \\
\hline $\mathrm{E}^{\prime}[\mathrm{cm} / \mathrm{s}]$ & $15 \pm 3$ & $14 \pm 4$ & 0.2 \\
\hline$A^{\prime}[\mathrm{cm} / \mathrm{s}]$ & $12 \pm 3$ & $12 \pm 4$ & 0.6 \\
\hline$E^{\prime} / A^{\prime}$ & $1.3 \pm 0.5$ & $1 \pm 0.4$ & 0.005 \\
\hline $\mathrm{SM}[\mathrm{cm} / \mathrm{s}]$ & $12 \pm 2.7$ & $11 \pm 2$ & 0.12 \\
\hline $\mathrm{ET}^{\prime}[\mathrm{cm} / \mathrm{s}]$ & $288 \pm 24$ & $294 \pm 27$ & 0.3 \\
\hline$E / E^{\prime}$ & $4.62(3.9-5.6)$ & $5.33(4.56-5.91)$ & 0.037 \\
\hline FMD [\%] & $11.64 \pm 3.4$ & $6.96 \pm 2.5$ & $<0.001$ \\
\hline $\mathrm{EFT}[\mathrm{cm}]$ & $0.28(0.20-0.32)$ & $0.31(0.24-0.35)$ & 0.04 \\
\hline
\end{tabular}

Data are mean \pm standard deviation (SD) or median interquartile range (IQR). LA — left atrium diameter; IVS — interventricular septal thickness; PW - posterior wall thickness; LVEDD - left ventricular end-diastolic diameter; LVESD - left ventricular end-systolic diameter; EF ejection fraction; E - early diastolic peak flow velocity; A — late diastolic peak flow velocity; DT — E-wave deceleration time; IVRT - isovolumic relaxation time; IVCT — isovolumic contraction time; ET — ejection time; $\mathrm{E}^{\prime}$ - early diastolic mitral annular velocity; $\mathrm{A}^{\prime}$ — late diastolic mitral annular velocity; SM - mitral annular systolic motion velocity; ET' - mitral annular ejection time; FMD - flow mediated dilatation; EFT - epicardial fat thickness

Table 3. Correlation analysis between chemerin and variables.

\begin{tabular}{lcc}
\hline Variables & $\mathbf{r}$ & $\mathbf{p}$ \\
\hline Age & 0.249 & $\mathbf{0 . 0 1 9}$ \\
Body mass index & 0.232 & $\mathbf{0 . 0 4 7}$ \\
Systolic BP & 0.264 & $\mathbf{0 . 0 2 3}$ \\
Diastolic BP & 0.378 & $\mathbf{0 . 0 0 1}$ \\
Waist circumference & 0.308 & $\mathbf{0 . 0 0 9}$ \\
E' $^{\prime}$ & -0.286 & $\mathbf{0 . 0 1 3}$ \\
E'/A' $^{\prime}$ & -0.272 & $\mathbf{0 . 0 2 6}$ \\
E/E' & 0.257 & $\mathbf{0 . 0 2 7}$ \\
EFT & 0.303 & $\mathbf{0 . 0 1 7}$ \\
FMD & -0.296 & $\mathbf{0 . 0 2 5}$ \\
\hline
\end{tabular}

$\mathrm{BP}$ - blood pressure; $\mathrm{E}$ - early diastolic peak flow velocity; $\mathrm{E}^{\prime}-$ early diastolic mitral annular velocity; $\mathrm{A}^{\prime}$ - late diastolic mitral annular velocity; EFT — epicardial fat thickness; FMD — flow mediated dilatation

and blood pressure, and with obesity and diabetes mellitus [23, 24]. Despite the fact that atheroscle- rosis risk factors were excluded from this study, to prevent them from being potential confounders, serum chemerin levels were found to be higher in psoriatic patients. In addition, this study results demonstrated a strong association between serum chemerin levels and age, BMI, SBP, DBP, and waist circumference.

Biyik et al. [25] identified the presence of LV diastolic dysfunction with mitral valve inflow measurements in psoriatic patients. However, in their study, blood pressure levels were significantly higher in psoriatic patients than in controls. One of the most common causes of diastolic dysfunction is hypertension. The present study found no significant differences in SBP and DBP between the two groups, and no LV diastolic dysfunction based on measurements of mitral valve inflow in the psoriatic patients. While the $\mathrm{E}$ wave velocity is dependent on preload and LA pressure, the $\mathrm{E}$ ' wave velocity is a reliable index of $\mathrm{LV}$ relaxation $[26,27]$. Furthermore, the E/E' ratio shows a better correlation with pulmonary capillary wedge pres- 
Table 4. Multivariate linear regression analysis with dependent variable $E / E^{\prime}$.

\begin{tabular}{lccc}
\hline Variable & Standardized $\beta$-coefficient & $95 \% \mathrm{Cl}$ & $\mathbf{p}$ \\
\hline Age & 0.459 & 0.021 to 0.110 & $\mathbf{0 . 0 0 4}$ \\
Systolic BP & 0.287 & -0.001 to 0.078 & 0.058 \\
Diastolic BP & -0.137 & -0.101 to 0.041 & 0.404 \\
Chemerin & 0.351 & 0.001 to 0.017 & $\mathbf{0 . 0 2 6}$ \\
Glocose & 0.012 & -0.031 to 0.034 & 0.932 \\
Waist circumference & -0.121 & -0.066 to 0.031 & 0.478 \\
LDL cholesterol & 0.079 & -0.010 to 0.017 & 0.589 \\
\hline
\end{tabular}

$\mathrm{Cl}$ - confidence interval; $\mathrm{BP}$ — blood pressure; LDL — low-density lipoprotein

sure than standard measurements of mitral valve inflow [28]. In the present study, the E/E' ratio in the psoriatic patients was significantly higher than in controls. Similarly, Bülbül Sen et al. [5] found a higher $\mathrm{E} / \mathrm{E}$ ' ratio in their psoriasis group. In addition, the present study found that serum chemerin levels correlated positively with the E/E' ratio and negatively with the E' and E'/A' ratios. Although the present study excluded conventional CVD risk factors to better evaluate the relationship between chemerin levels and diastolic function, it did use linear regression analysis, with age, SBP, DBP, waist circumference, glucose, and LDL-C as the independent variables. The results showed that in the psoriatic patients, chemerin levels and age were related to diastolic dysfunction independently from other risk factors. The association between chemerin and diastolic dysfunction may be explained by the chronic inflammatory environment of psoriasis. Weigert et al. [29] suggested that elevated serum chemerin was strongly related to CRP. Previous reports have shown that CRP decreases the production of nitric oxide and upregulates the expression of angiotensin type- 1 receptor, which is associated with endothelial dysfunction [30, 31]. Osto et al. [32] showed that patients with severe psoriasis showed early impairment of coronary microvascular function, which has also been reported to correlate with higher hs-CRP [33]. All of these factors could lead to diastolic dysfunction in psoriasis.

Endothelial dysfunction has been recognized as an early marker of subclinical atherosclerosis. FMD measurements are commonly used to evaluate endothelial function, and Balci et al. [34] showed that psoriatic patients had lower FMD values than normal controls. Gonzalez-Juanatey et al. [35] also demonstrated that the FMD percentage was lower in psoriatic patients. Similarly, the present study found that psoriatic patients had impaired FMD compared to controls. This study also showed that serum chemerin was strongly associated with impaired FMD.

Epicardial fat tissue is considered a metabolically active endocrine organ that secretes a number of pro-inflammatory and pro-atherogenic cytokines [12]. Prior studies have demonstrated associations between EFT and CVD, insulin resistance, coronary calcium score, and metabolic syndrome $[13,36]$. Previous studies have also reported that psoriatic patients had higher EFT values [16, 37]. The present study found higher EFT values in psoriatic patients than in healthy controls, which agrees with the results of previous studies. In addition, the present study found that serum chemerin correlated positively with EFT.

\section{Conclusions}

Cardiovascular morbidity and mortality are very common in psoriatic patients. However, early diagnosis and treatment of patients at high risk for CVD reduces mortality rates. The present study found that chemerin has a significant relationship with EFT and FMD, which are early signs of subclinical atherosclerosis. Also, the serum chemerin level is a marker of diastolic dysfunction in psoriatic patients. A major limitation of this study was the small number of patients enrolled. The results should be further analyzed with cause-and-effect studies that investigate chemerin's interactions with atherosclerosis. In addition, large population studies are needed to prove the study's hypothesis.

Funding: Istanbul Medeniyet University Research Fund supported this study (project number TSG-2013-333, TSA-2014-482).

\section{Conflict of interest: None declared}




\section{References}

1. Zabel BA, Allen SJ, Kulig P, et al. Chemerin activation by serine proteases of the coagulation, fibrinolytic, and inflammatory cascades. J Biol Chem. 2005; 280(41): 34661-34666, doi: 10.1074/ jbc.M504868200, indexed in Pubmed: 16096270.

2. Bozaoglu K, Bolton K, McMillan J, et al. Chemerin is a novel adipokine associated with obesity and metabolic syndrome. Endocrinology. 2007; 148(10): 4687-4694, doi: 10.1210/en.2007-0175, indexed in Pubmed: 17640997.

3. Albanesi C, Scarponi C, Pallotta S, et al. Chemerin expression marks early psoriatic skin lesions and correlates with plasmacytoid dendritic cell recruitment. J Exp Med. 2009; 206(1): 249-258, doi: 10.1084/jem.20080129, indexed in Pubmed: 19114666.

4. Gelfand JM, Weinstein R, Porter SB, et al. Prevalence and treatment of psoriasis in the United Kingdom: a population-based study. Arch Dermatol. 2005; 141(12): 1537-1541, doi: 10.1001/ archderm.141.12.1537, indexed in Pubmed: 16365254.

5. Bülbül Şen B, Ekiz Ö, Rifaioğlu EN, et al. Assessment of subclinical left ventricular dysfunction in patients with psoriasis by speckle tracking echocardiography: A Speckle Tracking Study. Int J Dermatol. 2016; 55(2): 158-164, doi: 10.1111/ijd.12703, indexed in Pubmed: 26104012.

6. Gullu H, Caliskan M, Dursun R, et al. Impaired coronary microvascular function and its association with disease duration and inflammation in patients with psoriasis. Echocardiography. 2013; 30(8): 912-918, doi: 10.1111/echo.12156, indexed in Pubmed: 23488894.

7. Mancini GB. The fire within psoriatic patients: overlap between inflammatory and cardiovascular diseases. Can J Cardiol. 2015; 31(3): 242-243, doi: 10.1016/j.cjca.2014.11.013, indexed in Pubmed: 25592850.

8. Shang Q, Tam LS, Yip GWK, et al. High prevalence of subclinical left ventricular dysfunction in patients with psoriatic arthritis. J Rheumatol. 2011; 38(7): 1363-1370, doi: 10.3899/ jrheum.101136, indexed in Pubmed: 21459943.

9. Barutcu A, Aksu F, Ozcelik F, et al. Evaluation of early cardiac dysfunction in patients with systemic lupus erythematosus with or without anticardiolipin antibodies. Lupus. 2015; 24(10): 1019-1028, doi: 10.1177/0961203315570164, indexed in Pubmed: 25697770.

10. Yu CM, Sanderson JE, Marwick TH, et al. Tissue Doppler imaging a new prognosticator for cardiovascular diseases. J Am Coll Cardiol. 2007; 49(19): 1903-1914, doi: 10.1016/j.jacc.2007.01.078, indexed in Pubmed: 17498573.

11. Deanfield J, Donald A, Ferri C, et al. Working Group on Endothelin and Endothelial Factors of the European Society of Hypertension. Endothelial function and dysfunction. Part I: Methodological issues for assessment in the different vascular beds: a statement by the Working Group on Endothelin and Endothelial Factors of the European Society of Hypertension. J Hypertens. 2005; 23(1): 7-17, indexed in Pubmed: 15643116.

12. Iacobellis G, Barbaro G. The double role of epicardial adipose tissue as pro- and anti-inflammatory organ. Horm Metab Res. 2008; 40(7): 442-445, doi: 10.1055/s-2008-1062724, indexed in Pubmed: 18401833.

13. Gorter PM, de Vos AM, van der Graaf Y, et al. Relation of epicardial and pericoronary fat to coronary atherosclerosis and coronary artery calcium in patients undergoing coronary angiography. Am J Cardiol. 2008; 102(4): 380-385, doi: 10.1016/j. amjcard.2008.04.002, indexed in Pubmed: 18678291.
14. Ding J, Hsu FC, Harris TB, et al. The association of pericardial fat with incident coronary heart disease: the Multi-Ethnic Study of Atherosclerosis (MESA). Am J Clin Nutr. 2009; 90(3): 499-504, doi: 10.3945/ajcn.2008.27358, indexed in Pubmed: 19571212.

15. Fang Na, Jiang M, Fan Yu. Association between psoriasis and subclinical atherosclerosis: A meta-analysis. Medicine (Baltimore). 2016; 95(20): e3576, doi: 10.1097/MD.0000000000003576, indexed in Pubmed: 27196459.

16. Balci A, Celik M, Balci DD, et al. Patients with psoriasis have an increased amount of epicardial fat tissue. Clin Exp Dermatol. 2014; 39(2): 123-128, doi: 10.1111/ced.12216, indexed in Pubmed: 24164295 .

17. Potenza C, Raimondi G, Pampena R, et al. Cardiovascular risk evaluation through heart rate variability analysis in psoriatic patients before and after 24 weeks of etanercept therapy: Prospective study. J Int Med Res. 2016; 44(1 suppl): 43-47, doi: 10.1177/0300060515593242, indexed in Pubmed: 27683138.

18. Ramsay B, Lawrence CM. Measurement of involved surface area in patients with psoriasis. Br J Dermatol. 1991; 124(6): 565-570, indexed in Pubmed: 2064940.

19. Keles N, Dogan B, Kalcik M, et al. Is serum Klotho protective against atherosclerosis in patients with type 1 diabetes mellitus? J Diabetes Complications. 2016; 30(1): 126-132, doi: 10.1016/j. jdiacomp.2015.09.013, indexed in Pubmed: 26601789.

20. Keles N, Caliskan M, Dogan B, et al. Low serum level of Klotho is an early predictor of atherosclerosis. Tohoku J Exp Med. 2015; 237(1): 17-23, doi: 10.1620/tjem.237.17, indexed in Pubmed: 26289053.

21. Horreau C, Pouplard C, Brenaut E, et al. Cardiovascular morbidity and mortality in psoriasis and psoriatic arthritis: a systematic literature review. J Eur Acad Dermatol Venereol. 2013; 27 Suppl 3: 12-29, doi: 10.1111/jdv.12163, indexed in Pubmed: 23845149.

22. Proietti I, Raimondi G, Skroza N, et al. Cardiovascular risk in psoriatic patients detected by heart rate variability (HRV) analysis. Drug Dev Res. 2014; 75 Suppl 1: S81-S84, doi: 10.1002/ ddr.21204, indexed in Pubmed: 25381987.

23. Han J, Kim SoH, Suh YJu, et al. Serum chemerin levels are associated with abdominal visceral fat in type 2 diabetes. J Korean Med Sci. 2016; 31(6): 924-931, doi: 10.3346/jkms.2016.31.6.924, indexed in Pubmed: 27247502.

24. Lehrke M, Becker A, Greif M, et al. Chemerin is associated with markers of inflammation and components of the metabolic syndrome but does not predict coronary atherosclerosis. Eur J Endocrinol. 2009; 161(2): 339-344, doi: 10.1530/EJE-09-0380, indexed in Pubmed: 19497986.

25. Biyik I, Narin A, Bozok MA, et al. Echocardiographic and clinical abnormalities in patients with psoriasis. J Int Med Res. 2006; 34(6): 632-639, doi: 10.1177/147323000603400608, indexed in Pubmed: 17294995.

26. Choong CY, Abascal VM, Thomas JD, et al. Combined influence of ventricular loading and relaxation on the transmitral flow velocity profile in dogs measured by Doppler echocardiography. Circulation. 1988; 78(3): 672-683, indexed in Pubmed: 3409503.

27. Nagueh SF, Middleton KJ, Kopelen HA, et al. Doppler tissue imaging: a noninvasive technique for evaluation of left ventricular relaxation and estimation of filling pressures. J Am Coll Cardiol. 1997; 30(6): 1527-1533, indexed in Pubmed: 9362412.

28. Garcia MJ, Ares MA, Asher C, et al. An index of early left ventricular filling that combined with pulsed Doppler peak E velocity may estimate capillary wedge pressure. J Am Coll Cardiol. 1997; 29(2): 448-454, indexed in Pubmed: 9015003. 
29. Weigert J, Neumeier M, Wanninger J, et al. Systemic chemerin is related to inflammation rather than obesity in type 2 diabetes. Clin Endocrinol (Oxf). 2010; 72(3): 342-348, doi: 10.1111/j.13652265.2009.03664.x, indexed in Pubmed: 19558533.

30. Venugopal SK, Devaraj S, Yuhanna I, et al. Demonstration that C-reactive protein decreases eNOS expression and bioactivity in human aortic endothelial cells. Circulation. 2002; 106(12): 1439-1441, indexed in Pubmed: 12234944.

31. Wang $\mathrm{CH}$, Li SH, Weisel $\mathrm{RD}$, et al. C-reactive protein upregulates angiotensin type 1 receptors in vascular smooth muscle. Circulation. 2003; 107(13): 1783-1790, doi: 10.1161/01. CIR.0000061916.95736.E5, indexed in Pubmed: 12665485.

32. Osto E, Piaserico S, Maddalozzo A, et al. Impaired coronary flow reserve in young patients affected by severe psoriasis. Atherosclerosis. 2012; 221(1): 113-117, doi: 10.1016/j.atherosclerosis.2011.12.015, indexed in Pubmed: 22236480.

33. Coimbra S, Oliveira H, Reis F, et al. C-reactive protein and leucocyte activation in psoriasis vulgaris according to severity and therapy.J Eur Acad Dermatol Venereol. 2010; 24(7): 789-796, doi: 10.1111/j.14683083.2009.03527.x, indexed in Pubmed: 20002653.
34. Balci DD, Balci A, Karazincir S, et al. Increased carotid artery intima-media thickness and impaired endothelial function in psoriasis. J Eur Acad Dermatol Venereol. 2009; 23(1): 1-6, doi: 10.1111/j.1468-3083.2008.02936.x, indexed in Pubmed: 18702627 .

35. Gonzalez-Juanatey C, Llorca J, Llorca J, et al. Endothelial dysfunction in psoriatic arthritis patients without clinically evident cardiovascular disease or classic atherosclerosis risk factors. Arthritis Rheum. 2007; 57(2): 287-293, doi: 10.1002/ art.22530.

36. Wang CP, Hsu HL, Hung WC, et al. Increased epicardial adipose tissue (EAT) volume in type 2 diabetes mellitus and association with metabolic syndrome and severity of coronary atherosclerosis. Clin Endocrinol (Oxf). 2009; 70(6): 876-882, doi: 10.1111/j.1365-2265.2008.03411.x, indexed in Pubmed: 18778397.

37. Bulbul Sen B, Atci N, Rifaioglu EN, et al. Increased epicardial fat tissue is a marker of subclinical atherosclerosis in patients with psoriasis. Br J Dermatol. 2013; 169(5): 1081-1086, doi: 10.1111/ bjd.12569, indexed in Pubmed: 23909282. 